\title{
GABA and Glutamate in Children with Primary Complex Motor Stereotypies: An 'H-MRS Study at 7T
}

\author{
(D)A.D. Harris, (D).S. Singer, (D)A. Horska, (D)T. Kline, (D) M. Ryan, (DR.A.E. Edden, and (DE.M. Mahone
}

\begin{abstract}
BACKGROUND AND PURPOSE: Complex motor stereotypies are rhythmic, repetitive, fixed, purposeful but purposeless movements that stop with distraction. They can occur in otherwise normal healthy children (primary stereotypies) as well in those with autism spectrum disorders (secondary stereotypies). The underlying neurobiologic basis for these movements is unknown but is thought to involve cortical-striatal-thalamo-cortical pathways. To further clarify potential neurochemical alterations, gamma-aminobutyric acid (GABA), glutamate, glutamine, $\mathrm{N}$-acetylaspartate, and choline levels were measured in 4 frontostriatal regions by using ${ }^{1} \mathrm{H}$ MRS at 7T.
\end{abstract}

MATERIALS AND METHODS: A total of 18 children with primary complex motor stereotypies and 24 typically developing controls, ages 5-10 years, completed MR spectroscopy at 7T. Single voxel STEAM acquisitions from the anterior cingulate cortex, premotor cortex, dorsolateral prefrontal cortex, and striatum were obtained, and metabolites were quantified with respect to $\mathrm{Cr}$ by using LCModel.

RESULTS: The 7T scan was well tolerated by all the participants. Compared with the controls, children with complex motor stereotypies had lower levels of GABA in the anterior cingulate cortex (GABA/ $\mathrm{Cr}, P=.049$; GABA/Glu, $P=.051)$ and striatum (GABA/Cr, $P=.028$; GABA/Glu, $P=.0037$ ) but not the dorsolateral prefrontal cortex or the premotor cortex. Glutamate, glutamine, NAA, and Cho levels did not differ between groups in any of the aforementioned regions. Within the complex motor stereotypies group, reduced GABA to $\mathrm{Cr}$ in the anterior cingulate cortex was significantly associated with greater severity of motor stereotypies $(r=-0.59, P=.021)$.

CONCLUSIONS: These results indicate possible GABAergic dysfunction within corticostriatal pathways in children with primary complex motor stereotypies.

ABBREVIATIONS: $\mathrm{ACC}=$ anterior cingulate cortex; $\mathrm{CMS}=$ complex motor stereotypies; $\mathrm{DLPFC}=$ dorsolateral prefrontal cortex; GABA = gamma-aminobutyric acid; $G l n=$ glutamine; $G l u=$ glutamate; $P M C=$ premotor cortex

$\mathbf{M}_{\mathrm{i}}^{\mathrm{R}}$ $\mathrm{R}$ spectroscopy allows for the noninvasive assessment of tissue metabolite content. It recently became possible to perform such measurements at ultra-high field (7T), with the benefits of increased SNR and improved separation of metabolite

Received April 20, 2015; accepted after revision August 12.

From the Russell H. Morgan Department of Radiology and Radiological Science (A.D.H., A.H., R.A.E.E.), Department of Neurology (H.S.S., T.K.), Department of Pediatrics (H.S.S.), and Department of Psychiatry and Behavioral Sciences (E.M.M.), The Johns Hopkins University School of Medicine, Baltimore, Maryland; and Department of Neuropsychology (M.R., E.M.M.) and F.M. Kirby Center for Functional Brain Imaging (A.D.H., A.H., R.A.E.E.), Kennedy Krieger Institute, Baltimore, Maryland.

Supported by National Institutes of Health grants: IR21 MH092693, P41 EB015909, U54 079123, and UL1 RR025005 and the Nesbit-McMaster Foundation. The funding agencies were not involved in the design, data collection, data analysis, manuscript preparation, or publication design. The authors have stated that they had no interests that could be perceived as posing a conflict or bias.

Previously presented, in part, at: Annual Meeting of the Child Neurology Society, October 10-14, 2014; Columbus, Ohio; and Annual Meeting of the International Society for Magnetic Resonance in Medicine, May 10-16, 2014; Milan, Italy. signals. ${ }^{1-3}$ In particular, the overlapping signals of glutamate (Glu), the primary excitatory neurotransmitter, and glutamine (Gln), its metabolic precursor, can be differentiated and quantified at 7T. Gamma-aminobutyric acid (GABA), the primary inhibitory neurotransmitter, can also be resolved and quantified at 7T. To date, no previous studies applied ultra-high-field measurements in young pediatric populations ( $<10$ years of age).

Complex motor stereotypies (CMS) are rhythmic, repetitive, fixed (in fashion, form, amplitude, and location) movements that appear purposeful but are purposeless (ie, serve no obvious adaptive function or purpose) and stop with sensory stimulation or

Please address correspondence to Ashley D. Harris, PhD, Russell H. Morgan Department of Radiology and Radiological Science, Johns Hopkins University School of Medicine, 600 North Wolfe St, Park 367C, Baltimore, MD 21287; e-mail: ashley.harris2@ucalgary.ca

-* Indicates open access to non-subscribers at www.ajnr.org

http://dx.doi.org/10.3174/ajnr.A4547 
distraction. ${ }^{4}$ Motor stereotypies are defined clinically and are differentiated from tics by their patterned, coordinated, repetitive, rhythmic, and nonreflexive features, and developmental time course. ${ }^{5}$ Episodes (eg, bilateral flapping and/or waving arm or hand movements, rotating the hands, and fluttering fingers in front of the face) last for periods of seconds to minutes, occur in clusters, and are associated with periods of excitement, engrossment, fatigue, boredom, and stress. Often occurring many times per day, these movements can adversely impact social, academic, and cognitive function. ${ }^{6}$

Although previously thought to only be associated with intellectual disability, sensory deprivation, or autism spectrum disorders, CMS are now recognized to occur as a "primary" form in as many as 3\%-4\% of otherwise typically developing children. ${ }^{7}$ Primary CMS typically appear before the age of 3 years, have a persistent course, and respond poorly to pharmacologic intervention. ${ }^{8,9}$ Stereotypies are not better described as tics or compulsions, though some degree of overlap and co-occurrence with other disorders that affect frontostriatal brain systems (attention deficit/hyperactivity disorder, obsessive compulsory disorder, tic disorders, developmental coordination disorder) is common. ${ }^{10}$ Young children who present with CMS are at particular risk for social, cognitive, and academic difficulties. Earlier identification and a better understanding of the neurobiologic markers of CMS can potentially minimize the harmful impact of the disorder and lead to more targeted behavioral and pharmacologic treatments.

The underlying pathophysiologic mechanism of stereotypies has not been fully established; hypotheses range from psychological concerns to neurobiologic abnormalities. ${ }^{5,9,11}$ The absence of premotor movement-related cortical potentials in children with primary CMS during these stereotypic movements indicates that motor stereotypies are initiated by different neural mechanisms than voluntary, purposeful movements. ${ }^{12}$ Current research indicates that stereotypies, similar to many other movement disorders, likely involve cortico-striatal-thalamo-cortical circuits and their interconnecting brain regions. Distinct cortical-striatal pathways that support goal-directed (ventromedial prefrontal to caudate) and habitual (premotor to putamen) behaviors have been identified ${ }^{13}$ and the limbic circuit (anterior cingulate cortex [ACC] to ventral striatum) is also involved in motor control ${ }^{13}$ through its contributions to the anticipation of tasks, emotional, motivational, reward, attention, vigor, autonomic information, and impulse control. ${ }^{14}$ In primary CMS, preliminary volumetric imaging results indicate a decreased volume of the putamen-caudate, ${ }^{15}$ and recent findings from a deer mouse model of CMS demonstrate a loss of striatal volume with a particular reduction in GABAergic medium spiny neurons (H.S.S., oral communication, January 22, 2015).

Several neurotransmitters, including dopamine, Glu, GABA, serotonin, norepinephrine, acetylcholine, and opioids, have important roles in cortico-striatal-thalamo-cortical pathways. To date, there has been limited in vivo investigation into the link between specific neurotransmitter anomalies and motor symptoms in CMS. ${ }^{1} \mathrm{H}$-MRS provides an opportunity to measure the major excitatory (Glu) and inhibitory (GABA) neurotransmitters involved in cortico-striatal-thalamo-cortical circuits in vivo. Glu and Gln are not as well resolved at 3T (and lower field strengths)

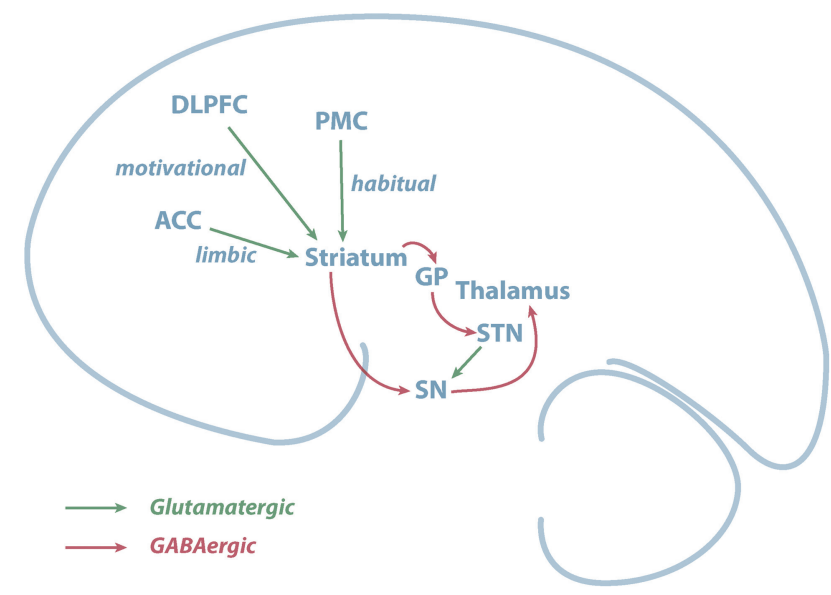

FIG 1. Illustration of the motor pathways and the neurotransmitter connections investigated in the current study. DLPFC, PMC, and ACC provide glutamatergic inputs to the striatum and outputs from the striatum to the globus pallidus and substantia nigra are GABAergic.

as at $7 \mathrm{~T}$ and, therefore, are often reported as Glx. This may be problematic because both of these amino acids can influence neuronal functions and there are pathologic processes in which one amino acid can increase and the other decrease. ${ }^{16}$ At higher field strengths (ie, 7T), Glu and Gln can more easily and reliably be differentiated. ${ }^{1,2}$ Previous research on habitual behaviors has primarily hypothesized altered dopaminergic neurotransmission, and animal models have been developed to mimic this change. ${ }^{17}$ Involvement of the glutamatergic system has been indicated based, in part, on the interaction between dopaminergic and glutamatergic activity in the corticostriatal system. ${ }^{13}$ In Tourette syndrome, another childhood movement disorder with intermittent paroxysmal motor activity, animal models and human studies have indicated a role for GABA. ${ }^{18-20}$

Potential glutamatergic and GABAergic dysfunction in the goal-directed, habitual, and limbic pathways (Fig 1) were the focus of the present study. Specifically, we hypothesized that GABA and Glu levels in these pathways would be altered in primary CMS compared with healthy controls, with the greatest dysfunction observed in regions that support habitual circuitry (premotorstriatal). Examination of Gln, NAA, and Cho was also completed to provide a contrast for examination of GABA and Glu.

\section{MATERIALS AND METHODS Participants}

This study was approved by the institutional review board of the Johns Hopkins Medical Institutions. Parents or legal guardians of all the participants provided informed, written consent, and all the participants provided assent before the study. Eighteen children diagnosed with nonautistic, primary CMS, age range 5-10 years, were recruited through the Pediatric Movement Disorders Clinic, Johns Hopkins Hospital; whereas 24 typically developing children were recruited through community advertisements to act as control participants. A pediatric neurologist (H.S.S.) made the diagnosis of CMS, with specific confirmation that movements were not better characterized as tics. Clinical characteristics used to distinguish motor stereotypies from chronic motor tics include the following: earlier age of onset, rhythmic movements of the upper extremities, a constant and fixed pattern of movements, a 
continuous and prolonged duration, the absence of a premonitory urge, stereotypy exacerbation when engrossed in an activity, and suppression by distraction (ie, calling one's name). ${ }^{13}$

Exclusion criteria included current Full Scale IQ $<80$, previous diagnoses of intellectual disability or autism spectrum disorders, and medication use in the past 3 months. In addition, all the participants in the CMS group were screened for autism spectrum disorders and excluded if they had a score of $>13$ when using the Autism Spectrum Screening Questionnaire. ${ }^{21}$ Severity of stereotypy symptoms was rated by parents at the time of scanning by using the Stereotypy Severity Scale. ${ }^{22}$ The Stereotypy Severity Scale is a 5-item caregiver questionnaire in which the parent rates the child's motor stereotypies with regard to the motor severity by rating the number, frequency, intensity, and interference of stereotypies (maximum, 18 points), and the global impairment (maximum, 50 points) during the past few days. Stereotypy Severity Scale motor and total stereotypy scores were used for brainbehavior analyses.

\section{MR Imaging and MR Spectroscopy Acquisition}

All the children had a mock scan training session to improve comfort, decrease anxiety, and train them to lie still. No sedation was used. Structural MRI and MRS were performed by using a 7T scanner (Achieva; Philips Healthcare, Best, the Netherlands) equipped with a quadrature transmit head coil and 32-channel receive coil array (Nova Medical, Wilmington, Massachusetts). A high-quality T1-weighted MPRAGE structural brain image (TE/ $\mathrm{TR}=2.1 / 4.8$ milliseconds; resolution, $0.6 \times 0.6 \times 0.6 \mathrm{~mm}^{3}$; total scan time, 6 minutes and 32 seconds) was acquired for planning of the MRS voxel locations and subsequent gray matter segmentation by using SPM (FIL Methods Group, UCL, London UK; http://www.fil.ion.ucl.ac.uk/spm/). ${ }^{23}$ Spectra were acquired from 4 voxels: ACC, dorsolateral prefrontal cortex (DLPFC), premotor cortex (PMC), and striatum. The STEAM sequence was used for signal localization with the following parameters: TE, mixing time, TR $=14$ milliseconds, 26 milliseconds, 3000 milliseconds, respectively, 96 averages, and VAPOR (variable power and optimized relaxation delays) water suppression. Voxel placement was performed by using a documented procedure and images for reference. The approximately 8-mL ACC voxel was placed in the left hemisphere on the ACC aligned tangential to the corpus callosum in the sagittal plane, with the posterior edge centered to the genu. This region of the ACC was selected to interrogate the premotorstriatal circuitry. The approximately 8-mL PMC voxel was placed on the left side, with the posterior face aligned to the precentral sulcus, and the inferior face above the level of the corpus callosum. The approximately 8-mL DLPFC voxel was placed on the left side, anterior to the premotor voxel, angulated so as to maximize GM content while avoiding the skull. The approximately 8 -mL striatum voxel was placed on the left side, aligned in the sagittal plane with the principal axis of the striatum.

\section{Postacquisition Image Analysis}

LCModel (http://www.lcmodel.com/) ${ }^{24}$ was used to quantify both GABA and Glu by using an in-house basis set that includes a macromolecular basis spectrum. GABA, Glu, Gln, NAA, and Cho were measured as a ratio to $\mathrm{Cr}$ within each voxel. GABA/Glu was also calculated to investigate inhibitory-excitatory balance.

\section{Statistical Analysis}

Age, socioeconomic status, handedness, and sex balance between the groups were compared by using ANOVAs for continuous variables and $\chi^{2}$ analyses for categoric variables. Distributions for metabolite ratios were examined for normality by using the Kolmogorov-Smirnov test, and those that showed significant skew were transformed by using square-root transformation (indicated in the text with superscript $\mathrm{t}$ ).

To examine global metabolite differences between the groups, all voxel locations were pooled and a linear mixed-effects model with the Fisher least significant difference as a post hoc test was used to examine differences in regional metabolite concentrations in CMS compared with controls, controlling for age, sex, and GM fraction within the voxel. This test examines differences in metabolite levels across all brain regions and benefits from an increase in power when examining the fixed effects factors (age and sex). Subsequently, to address the primary aim of the study, regional differences in metabolites were tested by using a second set of linear mixed-effects models for each voxel (ACC, DLPFC, PMC, and striatum). These models were derived and tested independently; however, regional measurements within a single participant are not independent. Within each region, measurements from different individuals are assumed to be independent. As such, no corrections for multiple comparisons were performed. The association between symptom severity (Stereotypy Severity Scale motor and total scores) and metabolite levels was examined by using Pearson correlations or partial correlations as applicable (controlling for GM fraction).

\section{RESULTS}

\section{Demographics}

Eighteen participants with CMS were recruited. One participant in the CMS group had comorbid diagnosis of separation anxiety disorder. The mean Autism Spectrum Screening Questionnaire score across subjects with CMS was $6.6 \pm 4.3$, with an inner quartile range of 3-9.75, well below the threshold for a diagnosis of comorbid autism spectrum disorder. There were no significant differences between CMS and control groups in age $(F[1,40]=$ $3.19, P=.082)$, socioeconomic status $(F[1,40]=0.379, P=.542)$, or handedness $\left(\chi^{2}=0.062, P=.094, n=42\right)$; however, there was a significant difference between groups in sex distribution $\left(\chi^{2}=\right.$ $7.41, P=.011, n=42)$. Most of the individuals in the CMS group $(14 / 18)$ and the control group (22/24) were right-handed, with no significant differences between groups in proportion of right- versus left-handers ( $\left.\chi^{2}=1.62, P=.375, n=42\right)$. For mixed-effects analyses that examined metabolite differences between groups, age, sex, and GM fraction were used as covariates in the model.

\section{MR Spectroscopy Acquisition}

The MR imaging and/or MR spectroscopy protocol of 45-60 minutes of duration was well tolerated in both controls and children with CMS. Nevertheless, all 4 MR spectroscopy voxels were not acquired in all the children due to movement or child fatigue, which resulted in some missing data. Two children (both con- 
trols) completed a second scanning session to acquire the remaining voxels. Table 1 summarizes the subject demographics for each voxel.

\section{Spectra}

Example spectra from a participant with CMS are shown in Fig 2. Across regions, the distributions for $\mathrm{GABA} / \mathrm{Cr}, \mathrm{Glu} / \mathrm{Cr}, \mathrm{Gln} / \mathrm{Cr}$, $\mathrm{NAA} / \mathrm{Cr}$, and Cho/Cr were significantly skewed (KolmogorovSmirnov test, $P<.05$ for each) and were thus transformed by using square-root transformation for subsequent analysis. The Kolmogorov-Smirnov tests confirmed the GABA/Glu data to be normally distributed. Due to the challenges of segmenting 7T pediatric images, GM fractions were available for the following 17/20; PMC, 17/19; striatum, 13/16; and for the CMS group: ACC, 16/16; DLPFC, 13/13; PMC, 12/13; and striatum, 18/18). To facilitate linear mixed-effects analyses, missing data for each voxel were imputed by using the mean GM fraction for the respective voxels.

To test for global group differences in metabolites (ie, across the whole brain, when controlling for age, sex, and GM fraction),

Table 1: Summary demographics for each voxel dataset

\begin{tabular}{lccccccc}
\hline & \multicolumn{3}{c}{ CMS } & & \multicolumn{3}{c}{ Controls } \\
\cline { 2 - 3 } & & $\begin{array}{c}\text { No. } \\
\text { Boys }\end{array}$ & $\begin{array}{c}\text { Age, } y \\
\text { (mean } \pm \text { SD) }\end{array}$ & & $\begin{array}{c}\text { No. } \\
\text { Boys }\end{array}$ & $\begin{array}{c}\text { Age, } y \\
\text { (mean } \pm \text { SD) }\end{array}$ \\
\hline ACC & 16 & 14 & $6.7 \pm 1.4$ & & 24 & 9 & $7.5 \pm 1.5$ \\
PMC & 13 & 10 & $6.4 \pm 1.5$ & & 18 & 9 & $7.4 \pm 1.4$ \\
Striatum & 18 & 15 & $6.7 \pm 1.5$ & & 17 & 9 & $7.1 \pm 1.2$ \\
DLPFC & 13 & 11 & $6.7 \pm 1.5$ & & 20 & 9 & $7.4 \pm 1.5$ \\
\hline
\end{tabular}
number of participants (for the controls: ACC, 21/24; DLPFC,

data were pooled across all regions and did not differ between groups for GABA/ $\mathrm{Cr}^{\mathrm{t}}(P=.119)$, Glu/ $\mathrm{Cr}^{\mathrm{t}}(P=.432)$, $\mathrm{Gln} / \mathrm{Cr}^{\mathrm{t}}$ $(P=.697), \mathrm{NAA} / \mathrm{Cr}^{\mathrm{t}}(P=.144)$, or $\mathrm{Cho} / \mathrm{Cr}^{\mathrm{t}}(P=.841)$. Conversely, the overall group difference for GABA/Glu approached significance $(P=.052)$. Although age and sex were not associated with any of these metabolite ratios across all voxels or within any individual voxel, they, nevertheless, were retained in the model. Across the whole brain, the GM fraction was associated significantly with Glu/Cr ${ }^{\mathrm{t}}$ and NAA/Cr ${ }^{\mathrm{t}}$ (both $P<.001$ ), and GABA/Glu $(P=.021)$, and was retained as a covariate for these models as well.

Data were subsequently analyzed in the individual voxels, controlling for age, sex, and GM fraction. Mean metabolite ratios within each voxel are presented in Table 2 . There were no significant group differences in metabolite levels in any of the 4 voxels for Glu/Cr ${ }^{\mathrm{t}}, \mathrm{Gln} / \mathrm{Cr}^{\mathrm{t}}$, NAA/Cr ${ }^{\mathrm{t}}$, or Cho/Cr ${ }^{\mathrm{t}}$. For GABA/Cr ${ }^{\mathrm{t}}$, there were significant metabolite reductions among the CMS group within the ACC $\left(F[1,35]=4.163, P=.049, \eta_{\mathrm{p}}{ }^{2}=0.106\right)$ and striatum $\left(F[1,30]=5.44, P=.027, \eta_{\mathrm{p}}{ }^{2}=0.154\right)$ but not within $\operatorname{DLPFC}\left(F[1,26]=0.526, P=.475, \eta_{\mathrm{p}}{ }^{2}=0.020\right)$ or PMC $(F[1,27]$ $\left.=2.156, P=.121, \eta_{\mathrm{p}}{ }^{2}=0.087\right)$. A similar pattern was seen for GABA/Glu, which showed reductions among the patients with CMS in ACC $\left(F[1,35]=4.07, P=.051, \eta_{\mathrm{p}}{ }^{2}=0.104\right)$ and striatum $\left(F[1,30]=4.74, P=.037, \eta_{\mathrm{p}}{ }^{2}=0.137\right)$, but not DLPFC $\left(F[1,26]=0.01, P=.958, \eta_{\mathrm{p}}{ }^{2}<0.001\right)$ or $\operatorname{PMC}(F[1,27]=1.48$, $\left.P=.234, \eta_{\mathrm{p}}{ }^{2}=0.052\right)$.

The association between GABA ratios $\left(\mathrm{GABA} / \mathrm{Cr}^{\mathrm{t}}, \mathrm{GABA} /\right.$ Glu) and stereotypy severity (Stereotypy Severity Scale) were examined in the CMS group within the voxels, showing group differences (ACC, striatum). Within the ACC, the GABA/Cr ${ }^{\mathrm{t}}$ ratio was significantly negatively associated
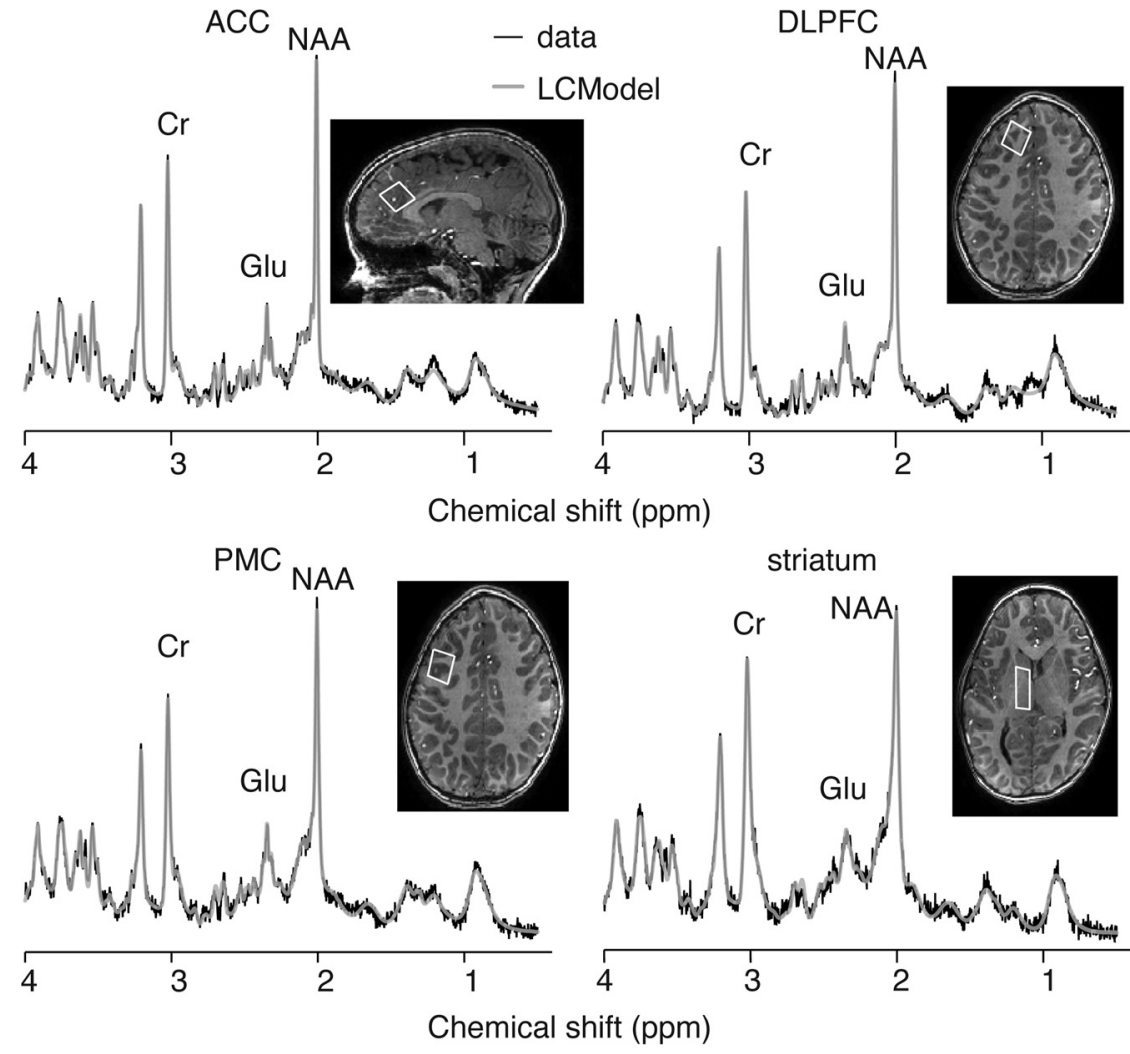

FIG 2. Example spectra and LCModel fit from each voxel. with the total motor Stereotypy Severity Scale $(r=-0.59, P=.021, n=15)($ Fig 3). Within the striatum, both $\mathrm{GABA} / \mathrm{Cr}^{\mathrm{t}}$ $(r=0.50, P=.039, n=17)$ and GABA/ Glu $(r=0.58, P=.019, n=15)$ showed significant positive associations with the total Stereotypy Severity Scale score.

\section{DISCUSSION}

To our knowledge, this is the first ${ }^{1} \mathrm{H}$-MRS study performed at $7 \mathrm{~T}$ in a young pediatric clinical population. Although the 7T MR system is classified as an investigational device, it is a nonsignificant-risk device per the FDA. Strict guidelines guard against excessive power deposition, which was continuously monitored, and the acquisitions used here stayed well within these limits. The combined experience at multiple institutions over the past 10 years in adults has not revealed any significant risks of 7T imaging. Some participants had experienced dizziness, and, for this reason, we do not recruit participants with a history of vertigo, seizure disorder, middle ear disorder, and double vision. Since 
Table 2: GABA and Glu levels across all voxels in the CMS and control groups

\begin{tabular}{|c|c|c|c|c|c|c|}
\hline & \multicolumn{2}{|c|}{$\mathrm{GABA} / \mathrm{Cr}$} & \multicolumn{2}{|c|}{$\mathrm{Glu} / \mathrm{Cr}$} & \multicolumn{2}{|c|}{ GABA/Glu } \\
\hline & CMS & Controls & CMS & Controls & CMS & Controls \\
\hline ACC & $0.21 \pm 0.052^{a}$ & $0.28 \pm 0.11^{a}$ & $1.65 \pm 0.10$ & $1.64 \pm 0.10$ & $0.13 \pm 0.029^{a}$ & $0.17 \pm 0.059^{a}$ \\
\hline DLPFC & $0.23 \pm 0.10$ & $0.22 \pm 0.20$ & $1.66 \pm 0.10$ & $1.60 \pm 0.16$ & $0.13 \pm 0.066$ & $0.13 \pm 0.10$ \\
\hline PMC & $0.23 \pm 0.13$ & $0.26 \pm 0.12$ & $1.60 \pm 0.13$ & $1.67 \pm 0.16$ & $0.14 \pm 0.077$ & $0.15 \pm 0.061$ \\
\hline Striatum & $0.24 \pm 0.055^{a}$ & $0.28 \pm 0.049^{a}$ & $1.29 \pm 0.12$ & $1.28 \pm 0.15$ & $0.18 \pm 0.038^{a}$ & $0.22 \pm 0.048^{a}$ \\
\hline
\end{tabular}

${ }^{a}$ Significant differences between $\mathrm{CMS}$ and control groups are indicated $(P<.05)$.

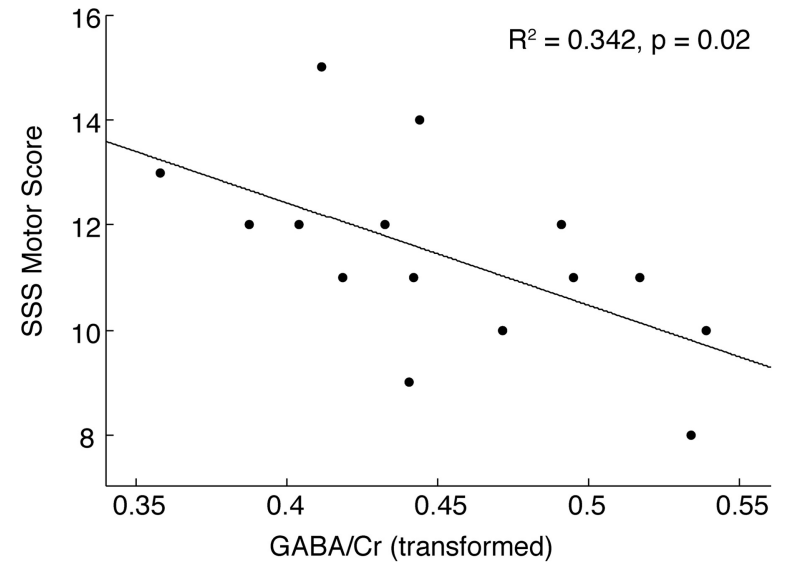

FIG 3. Association between GABA/Cr (after square-root transformation) in the ACC and severity of motor stereotypy.

2009 , our research team has scanned $>100$ children at $7 \mathrm{~T}$ with no adverse effects. The MR spectroscopy protocol here was well tolerated in all 42 participants, with no report of dizziness, nausea, or other discomfort. The objective was to investigate GABA and Glu in the corticostriatal networks thought to be involved in the neurobiology of CMS. ${ }^{13,25}$ The use of an ultra-high field affords increased SNR and spectra resolution, and enables the quantification of Glu rather than the mixed index of Glx. ${ }^{1-3}$

Although there is limited literature for comparison, the levels of GABA and Glu are consistent with a previous 7T study with a voxel in the ACC. ${ }^{3}$ For this study, $\mathrm{Cr}$ was used as the reference signal. The implicit assumption that $\mathrm{Cr}$ does not change across the brain or with development is supported by finding only GABA and GABA/Glu differences in 2 voxels and no changes in other metabolites. Although water-signal referencing is becoming the standard in MR spectroscopy, it was not possible in the current study, and it is not clear how water-referenced metabolite quantification would be impacted by changes in water relaxation during childhood. ${ }^{26,27}$ The primary findings were decreased GABA levels in CMS in the striatum and ACC but not in the PMC or DLPFC, and associations between ACC and striatal GABA and stereotypy severity. Similarly, decreases of GABA/Glu were detected in the ACC and striatum. These findings are likely driven by the decrease in GABA in these regions. No group differences in Glu, Gln, NAA, or Cho were detected. NAA and Cho analysis were included for completeness, not to investigate a specific hypothesis for these metabolites. This pattern of results indicates that inhibitory dysfunction in the ACC and striatum are associated with primary CMS. Of note, these measurements were obtained at rest, without accounting for the time from the last stereotypic behavior. Whether there are additional differences that present during a stereotypy episode cannot be quantified by using this methodology.

Reduced striatal GABA in young children with CMS was identified in this study. Unfortunately, the limited spatial resolution of MR spectroscopy does not allow differentiation among putamen (habitual pathway), caudate (goal-directed pathway), and ventral (limbic pathway) striatum (Fig 1). The habitual motor network, composed of the PMC and putamen, ${ }^{13}$ is involved in the development, learning, and control of habitual motor actions. ${ }^{28}$ Functional connectivity analysis implicated the habitual circuit in slips of action (ie, extraneous behavior directed at no longer rewarding outcomes), ${ }^{29}$ and a morphologic study detected reduced putamen volume in older children with CMS. ${ }^{10}$ Hence, the current data could support a central role for the habitual pathway in $\mathrm{CMS}^{4,11,13}$ based, in part, on decreased GABAergic inhibition either within or involving output from the putamen. Another small volumetric study in CMS identified a decrease in size and/or GM attenuation of the caudate, ${ }^{15}$ which raises the possibility of a dysfunction in the goal-directed pathway. Regardless of whether the goal-directed or habitual pathway is involved, decreased levels of GABA in the striatum indicate that inhibitory output from the striatum is altered in childhood CMS.

The observation of decreased GABA and its association with stereotypy severity in the ACC raises the possibility that a dysfunction in CMS could be within the emotional-limbic circuit. The emotional-limbic circuit includes the ACC projections to the olfactory tubercle and nucleus accumbens (ventral striatum), as shown in Fig 1. Whether the behavioral alteration is related directly to the ACC involvement in conflict monitoring and response $^{30}$ or is secondary to increased, but undetected, glutamatergic activity from the ACC to the ventral striatum is unknown.

The combined results of this study indicate that CMS is not associated with direct alterations in Glu levels in children. Nevertheless, decreased GABA levels could result in poor GABAergic modulation and tuning of glutamatergic synapses rather than simply impairing GABAergic network components. Although 7T MR spectroscopy provides the ability to more easily and reliably differentiate Glu from its precursor Gln, it does not provide the ability to differentiate the metabolic and synaptic pools of Glu or GABA. ${ }^{31}$ It, therefore, is possible that an imbalance between the metabolic and neurotransmitter Glu pools is present, which impacts glutamatergic function, but is not detected by MR spectroscopy. It is important to note that all statistical models were devised and tested independently. Measurements from different individuals are assumed to be independent; however, measurements from different regions within an individual are not independent. Thus, in this study, no corrections for multiple comparisons were applied, but significant results would not have survived a Bonferroni correction for multiple comparisons. 


\section{CONCLUSIONS}

To investigate the neurobiology of CMS, we successfully performed an MR spectroscopy study of children at 7T to examine GABA and Glu in multiple brain regions. We showed significantly lower GABA in the ACC and striatum in patients with CMS compared with healthy controls. These findings were consistent with abnormal function of corticostriatal networks in children with primary CMS.

Disclosures: Alena Horska—RELATED: Grant: National Institutes of Health (NIH),* Comments: Dr. H. Singer, Principal Investigator. Matthew Ryan-RELATED: Supported by 1R21 MH092693; the Nesbit-McMaster Foundation; U54 079123; and UL1 RR025005.* The funding agencies were not involved in the design, data collection, data analysis, manuscript preparation, or publication design. Richard EddenRELATED: Grant: This work was supported by NIH EB015909.* E. Mark MahoneRELATED: Grant: NIH 1R21 MH092693, , NIH P41 EB015909, * NIH U54 079123, * NIH UL1 RR025005, ${ }^{*}$ Nesbit-McMaster Foundation*; Support for Travel to Meetings for the Study or Other Purposes: NIH 1R21 MH092693. *Money paid to the institution.

\section{REFERENCES}

1. Tkac I, Oz G, Adriany G, et al. In vivo $1 \mathrm{H}$ NMR spectroscopy of the human brain at high magnetic fields: metabolite quantification at 4T vs. 7T. Magn Reson Med 2009;62:868-79 CrossRef Medline

2. Mekle R, Mlynárik V, Gambarota G, et al. MR spectroscopy of the human brain with enhanced signal intensity at ultrashort echo times on a clinical platform at 3T and 7T. Magn Reson Med 2009;61: 1279-85 CrossRef Medline

3. Dou W, Palomero-Gallagher N, van Tol MJ, et al. Systematic regional variations of GABA, glutamine, and glutamate concentrations follow receptor fingerprints of human cingulate cortex. $\mathrm{J} \mathrm{Neu}$ rosci 2013;33:12698-704 CrossRef Medline

4. Singer HS. Stereotypic movement disorders. Handb Clin Neurol 2011;100:631-39 CrossRef Medline

5. Mahone EM, Bridges D, Prahme C, et al. Repetitive arm and hand movements (complex motor stereotypies) in children. J Pediatr 2004;145:391-95 CrossRef Medline

6. McCurdy MD, Bellows A, Ferrand J, et al. Social cognition, repetitive behavior, and ADHD symptoms among children with primary complex motor stereotypies. J Int Neuropsychol Soc 2014;20:12

7. Foster LG. Nervous habits and stereotyped behaviors in preschool children. J Am Acad Child Adolesc Psychiatry 1998;37:711-17 CrossRef Medline

8. Harris KM, Mahone EM, Singer HS. Nonautistic motor stereotypies: clinical features and longitudinal follow-up. Pediatr Neurol 2008;38: 267-72 CrossRef Medline

9. Oakley C, Mahone EM, Morris-Berry C, et al. Primary complex motor stereotypies in older children and adolescents: clinical features and longitudinal follow-up. Pediatr Neurol 2015;52:398-403.e1 CrossRef Medline

10. Mahone EM, Crocetti D, Kline T, et al. Anomalous putamen development in children with primary complex motor stereotypies. Annals Neurol 2014;76(suppl 18):S227

11. Gao S, Singer HS. Complex motor stereotypies: an evolving neurobiological concept. Future Neurol 2013;8:273-85

12. Houdayer E, Walthall J, Belluscio BA, et al. Absent movement-related cortical potentials in children with primary motor stereotypies. Mov Disord 2014;29:1134-40 CrossRef Medline

13. Singer HS. Motor control, habits, complex motor stereotypies, and
Tourette syndrome. Ann N Y Acad Sci 2013;1304:22-31 CrossRef Medline

14. Gruber AJ, McDonald RJ. Context, emotion, and the strategic pursuit of goals: interactions among multiple brain systems controlling motivated behavior. Front Behav Neurosci 2012;6:50 CrossRef Medline

15. Kates WR, Lanham DC, Singer HS. Frontal white matter reductions in healthy males with complex stereotypies. Pediatr Neurol 2005;32: 109-12 CrossRef Medline

16. Novotny EJ Jr, Fulbright RK, Pearl PL, et al. Magnetic resonance spectroscopy of neurotransmitters in human brain. Ann Neurol 2003;54(suppl 6):S25-S31 CrossRef Medline

17. Aliane V, Perez S, Bohren Y, et al. Key role of striatal cholinergic interneurons in processes leading to arrest of motor stereotypies. Brain 2011;134:110-18 CrossRef Medline

18. Macri S, Onori MP, Roessner V, et al. Animal models recapitulating the multifactorial origin of Tourette syndrome. Int Rev Neurobiol 2013;112:211-37 CrossRef Medline

19. Puts NA, Harris AD, Crocetti D, et al. Reduced GABAergic inhibition and abnormal sensory processing in children with Tourette syndrome. J Neurophysiol 2015;114:808-17 CrossRef Medline

20. Draper A, Stephenson MC, Jackson GM, et al. Increased GABA contributes to enhanced control over motor excitability in Tourette syndrome. Curr Biol 2014;24:2343-47 CrossRef Medline

21. Ehlers S, Gillberg C, Wing L. A screening questionnaire for Asperger syndrome and other high-functioning autism spectrum disorders in school age children. J Autism Dev Disord 1999;29:129-41 CrossRef Medline

22. Miller JM, Singer HS, Bridges DD, et al. Behavioral therapy for treatment of stereotypic movements in nonautistic children. J Child Neurol 2006;21:119-25 CrossRef Medline

23. Ashburner J, Friston KJ. Unified segmentation. NeuroImage 2005;26: 839-51 CrossRef Medline

24. Provencher SW. Estimation of metabolite concentrations from localized in vivo proton NMR spectra. Magn Reson Med 1993;30: 672-79 CrossRef Medline

25. Balleine BW, O'Doherty JP. Human and rodent homologies in action control: corticostriatal determinants of goal-directed and habitual action. Neuropsychopharmacology 2010;35:48-69 CrossRef Medline

26. Kumar R, Delshad S, Macey PM, et al. Development of T2-relaxation values in regional brain sites during adolescence. Magn Reson Imaging 2011;29:185-93 CrossRef Medline

27. Paus T, Collins DL, Evans AC, et al. Maturation of white matter in the human brain: a review of magnetic resonance studies. Brain Res Bull 2001;54:255-66 CrossRef Medline

28. Tricomi E, Balleine BW, O'Doherty JP. A specific role for posterior dorsolateral striatum in human habit learning. Eur J Neurosci 2009; 29:2225-32 CrossRef Medline

29. de Wit S, Watson P, Harsay HA, et al. Corticostriatal connectivity underlies individual differences in the balance between habitual and goal-directed action control. J Neurosci 2012;32:12066-75 CrossRef Medline

30. Botvinick MM, Cohen JD, Carter CS. Conflict monitoring and anterior cingulate cortex: an update. Trends Cogn Sci 2004;8:539-46 CrossRef Medline

31. Rae CD. A guide to the metabolic pathways and function of metabolites observed in human brain $1 \mathrm{H}$ magnetic resonance spectra. Neurochem Res 2014;39:1-36 CrossRef Medline 